\title{
Development and Characterization of an Intraocular Biodegradable Polymer System Containing Cyclosporine-A for the Treatment of Posterior Uveitis
}

\author{
Juliana Barbosa Saliba a, André Augusto Gomes Faraco, Maria Irene Yoshida ${ }^{\mathrm{b}}$, \\ Wander Luiz de Vasconcelos ${ }^{c}$, Armando da Silva-Cunha ${ }^{\mathrm{a}}$,Herman Sander Mansur ${ }^{c *}$ \\ ${ }^{a}$ Departamento de Produtos Farmacêuticos, Universidade Federal de Minas Gerais - UFMG, \\ Av. Antônio Carlos, 6627, 31270-010 Belo Horizonte - MG, Brazil \\ ${ }^{\mathrm{b}}$ Departamento de Química, Universidade Federal de Minas Gerais - UFMG \\ Av. Antônio Carlos, 6627, 31270-010 Belo Horizonte - MG, Brazil \\ ${ }^{\mathrm{c}}$ Departamento de Engenharia Metalúrgica e de Materiais, \\ Universidade Federal de Minas Gerias - UFMG, \\ Rua Espírito Santo, 35/316, Centro, 30160-030 Belo Horizonte - MG, Brazil
}

Received: December 4, 2007; Revised: February 29, 2008

\begin{abstract}
The aim of this study was to synthesize and characterize the biodegradable intraocular implants based on poly (D,L-lactide-co-glycolide) (PLGA 75:25) with Cyclosporine-A (CyA) and to evaluate their in vitro drug delivery profile. Thermal analysis was conducted by using Thermogravimetry (TG) and Differential Scanning Calorimetry (DSC). Phase analysis and crystallinity of the polymer-CyA samples were assessed through X ray diffraction (XRD) and Fourier transform infrared spectroscopy (FTIR). Finally, microstructure and morphology of the systems were investigated by Scanning Electron Microscopy (SEM). The results showed that CyA was successfully incorporated into PLGA network with drug loading of approximately $31.6 \%$. Also, based on FTIR and thermal analyses (TGA/DSC) no significant physical-chemical interaction was detected at the micro-nanoscale level between polymer/drug. SEM micrographs have indicated a uniform drug distribution in PLGA matrix. XRD patterns have showed that the incorporated semi-crystalline structure of CyA has not significantly altered the polymeric mainly amorphous network. In addition, the results have confirmed the chemical and biological drug stability, the drug distribution into the polymeric matrix and the possibility of cyclosporine prolonged delivery system profile.
\end{abstract}

Keywords: nanostructured drug delivery system, biodegradable intraocular implants, biodegradable polymer

\section{Introduction}

Biodegradable polymers have been largely used in pharmaceuticals and biomedical field, respectively as delivery systems or as biomaterials ${ }^{1}$. The poly (D,L-lactide-co-glycolide) is a classic example amongst the synthetic polymers and has been well applied as drug delivery system due to its satisfactory biocompatibility and absence of significant toxicity in vivo studies ${ }^{2,3}$. Due to biodegradable intraocular implants nanoparticle constitution, the nanoscience can be an important tool to study their properties based on the polymer/ drug interaction analysis.

The drug/polymers interface has been previously reported and characterized by conformational, spectroscopic, thermal and morphological techniques in order to detect possible polymer and drug interaction and to justify the preliminary in vitro release profile. The PLGA 75:25, presents linear chain containing 75\% glucolide and $25 \%$ lactide. The lactide differs from glycolide by the presence of methyl group, characterizing its higher hydrophobicity. The polymer proportion and the chain size will define the in vitro and in vivo copolymer degradation. Besides that, this copolymer presents an amorphous state and its glass transition temperature occurs at, approximately $50{ }^{\circ} \mathrm{C}$. This material also presents thermoplastic property where

*e-mail: hmansur@demet.ufmg.br

Article presented at the II Simpósio Mineiro de Ciências dos Materiais

November 12-14, 2007, Ouro Preto - MG. copolymer chains are held together by relatively weak van der Waals and dipole-dipole forces ${ }^{1}$.

The CyA is a cyclic endecaptide commonly used as a selective immunosuppressive drug, with anti-inflammatory properties effective in the treatment of the posterior uveitis isolated from fungal. The lyophilized drug exhibits characteristics consisted with a glass thermotropic liquid in a semi-crystalline state and has solid-liquid phase transition in range of $118-125^{\circ} \mathrm{C}^{11}$. The presence of $\beta$-sheets intermolecular interaction indicates the molecular stability and biological activity was maintained ${ }^{4}$. Cyclosporine A has been widely and effectively used for the treatment of various forms of chronic uveitis. However, poor penetration of topical CsA into the eye and serious systemic toxicity produced by oral administration require a new approach to overcome the hurdles of blood-ocular block and low bioavailability of $\mathrm{CyA}^{12}$.

In the present work, our main goal was the development and characterization of an Intraocular Biodegradable Polymer System based on poly(D,L-lactide-co-glycolide) (PLGA 75:25) and the Cyclosporine $\mathrm{A}$ (CyA), so it will be possible to replace the conventional forms of systemic drug administration for the treatment of one important group of ocular disease, posterior uveitis. 


\section{Experimental Procedure}

\subsection{Preparation of the intraocular implants}

The intraocular implants were prepared by molding a lyophilized mixture consisted of a homogeneous mass containing $75 \%$ of poly(D,L-lactide-co-glycolide, in ratio of 75:25, called PLGA $75: 25)$ and $25 \%$ of cyclosporine A (CyA) into rods using a Teflon ${ }^{\circledR}$ sheet heated on a hot plate at a temperature from 100 to $120{ }^{\circ} \mathrm{C}$. The lyophilized mixture was obtained by dissolving PLGA 75:25 and cyclosporine $\mathrm{A}(\mathrm{CyA})$ in a adequate quantity of acetonitrile as organic solvent $\left(\mathrm{CH}_{3} \mathrm{CN}\right)$. The formed solution was then lyophilized during 24/48 hours.

\section{2. $X$ ray diffraction analysis (XRD)}

The lyophilized samples PLGA 75:25, CyA and lyophilized mixture was analyzed by (PHILIPS, PW1710) using $\mathrm{CuK} \alpha$ radiation with $\lambda=1.54056 \AA$. XRD analyses were conducted in the $2 \theta$ range from 3.03 to $89.91^{\circ}$ with steps of $0.06^{\circ}$. Narrow peaks identified within the scan range were confirmed using previously published literature ${ }^{9}$.

\subsection{Thermogravimetry characterization $(T G)$}

The thermal stability was evaluated by thermogravimetry analysis (TG, Shimatzu TGA 50H). The lyophilized samples PLGA 75:75 and $\mathrm{CyA}$ were heated in sealed platinum pans at a heating rate of $10{ }^{\circ} \mathrm{C} . \mathrm{min}^{-1}$ from room temperature to $750{ }^{\circ} \mathrm{C}$ under nitrogen atmosphere at rate of $50 \mathrm{~mL} \cdot \mathrm{min}^{-1}$.

\subsection{Differential scanning calorimetry characterization (DSC)}

To evaluate the possible interaction between the compounds, the thermal analysis was performed by differential scanning calorimetry (DSC, Shimatzu DSC-50). The samples CyA, PLGA 75:25 and lyophilized mixture was heated in sealed aluminum pans, and the first scan was measured at a heating rate of $10^{\circ} \mathrm{C} \cdot \mathrm{min}^{-1}$ from 100 to $180{ }^{\circ} \mathrm{C}$ under helium atmosphere. Calibration of the system was performed using indium standard.

\subsection{Fourier transform infrared spectroscopy characterization (FTIR)}

Fourier transform infrared spectroscopy (FTIR) characterization of pure PLGA 75:25 and CyA mixture in ratio of 100:10 and the lyophilized mixture was conducted in order to investigate the presence of specific chemical groups and interactions between the components. FTIR spectra were obtained within the range between 4000 and $400 \mathrm{~cm}^{-1}$ (Perkin-Elmer, Paragon 1000), using the diffuse reflectance spectroscopy method (DRIFTS-FTIR). Samples were mixed with dried $\mathrm{KBr}$ powder $(1.0 \mathrm{wt} \%)$, then placed in a sampling cup and 64 scans were acquired at $2 \mathrm{~cm}^{-1}$ resolution with the subtraction of $\mathrm{KBr}$ background.

\subsection{The preliminary in vitro study}

The preliminary in vitro study was conducted by placing a tube containing $2.0 \mathrm{~mL}$ of BSS (balanced salt solution, Bausch \& Lomb) and the implant was placed in an incubator at $37^{\circ} \mathrm{C}$ at $30 \mathrm{rpm}$. At predetermined intervals, the entire medium was sampled and other $2.0 \mathrm{~mL}$ of fresh medium was immediately added to each tube. The release profile was evaluated as a cumulative percentage of cyclosporine-A released in the medium. The amount of the drug released was measured by high-performance liquid chromatography (HPLC) using a Waters $^{\circledR}$ apparatus.

\subsection{Scanning electron microscopy characterization (SEM)}

The implant sample morphology scaffolds was analyzed by SEM (JEOL model JSM-6360LV). Prior to SEM analysis samples were gold-coated, then directly observed at an accelerating voltage of $15 \mathrm{KV}$.

\section{Results and Discussion}

\subsection{Synthesis and preparation of the intraocular implants}

Figure 1 shows the mixture lyophilized implants characteristics which presented cylindrical shape with approximately $4.00 \mathrm{~mm}$ of length and $1.00 \mathrm{~mm}$ of diameter. The average weight of implants was $5.9 \pm 0.1 \mathrm{mg}(\mathrm{n}=10)$ and the average incorporation of CyA into the polymeric matrix was of $31.6 \%(n=3)$.

In addition, SEM micrographs of PLGA-CyA lyophilized samples showed a morphological aspect of uniform and homogeneous surface and the absence of significant superficial irregularities (Figure 2a,b). Hence, the micrographs have proven to be an important tool for an adequate understanding the implant under in vitro delivery profile.

\subsection{The $X$ ray diffraction}

Figure 3 shows the XRD results of lyophilized mixture and lyophilized samples of CyA and PLA 75:25.The peaks between $7^{\circ}$ and $4^{\circ}$ (2è) correspond to the CyA semi-crystalline state (Figure $3 a)^{8}$ and no peak has been observed for the PLGA 25:25 (Figure $3 b$ ). Therefore, the results confirmed its amorphous state ${ }^{9}$. In addition, the crystallinity of lyophilized CyA (Figure 3a) was not observed in the lyophilized mixture sample (Figure $3 \mathrm{c}$ ). This fact can be explained by the possibility of the CyA distribution into the polymeric matrix. It is believed that the drug presents a small molecular

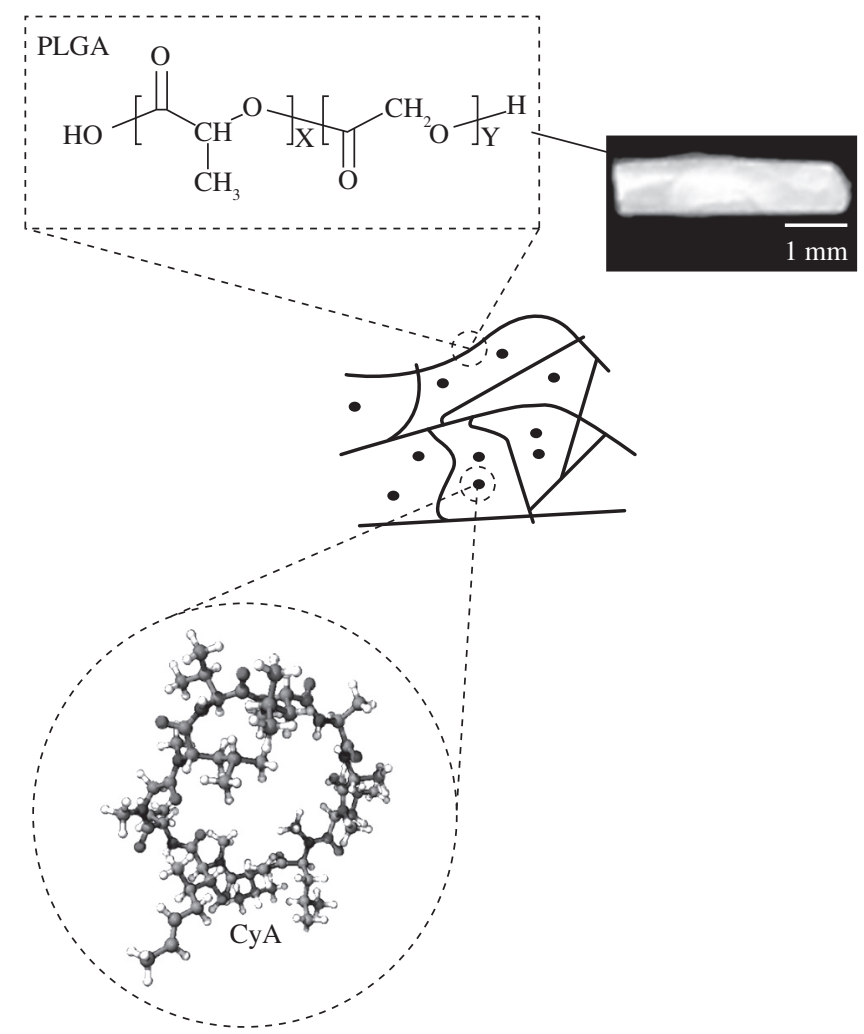

Figure 1. Picture of the developed implants from the mixture lyophilized. 


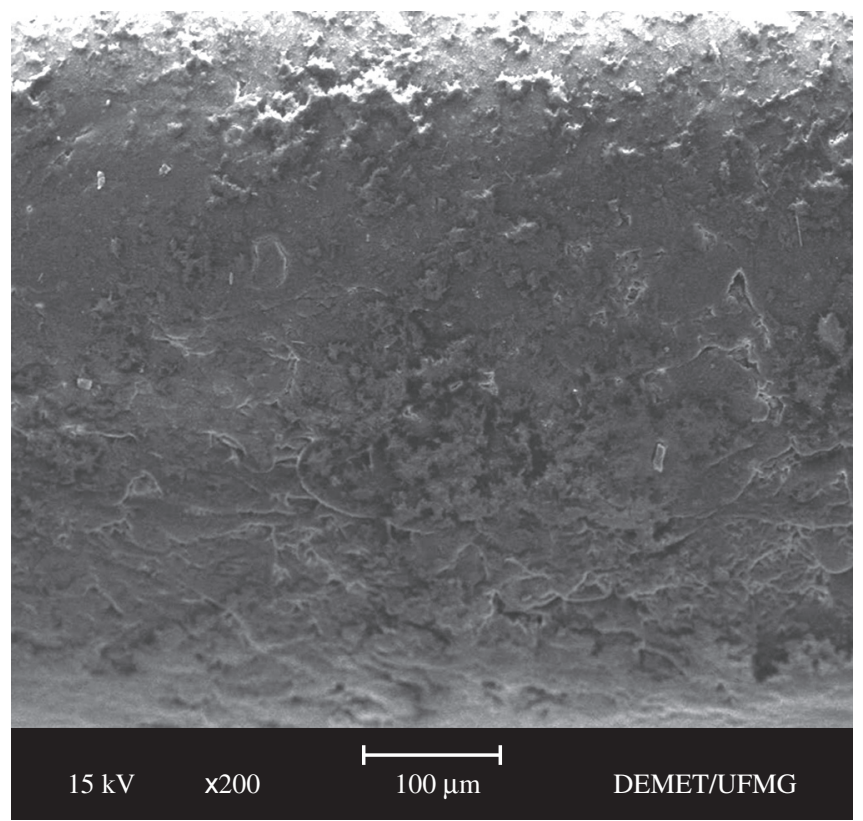

(a)

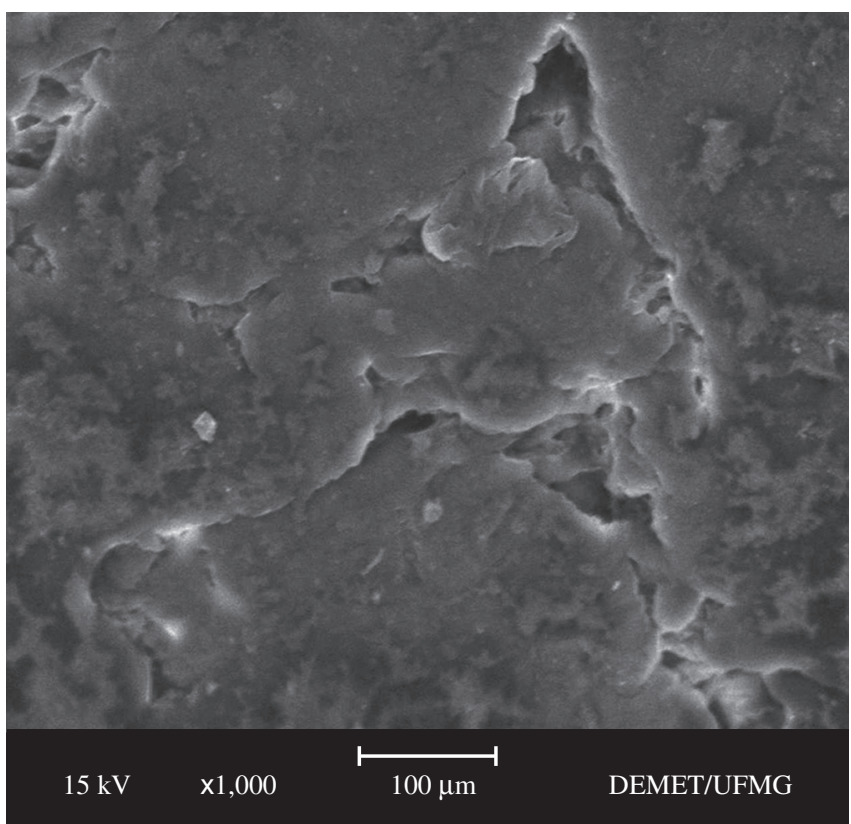

(b)

Figure 2. SEM pictures from the mixture lyophilized implant before in vitro incubation a) magnification 200x; b) magnification 1,000x, with just minor porosity at the surface.

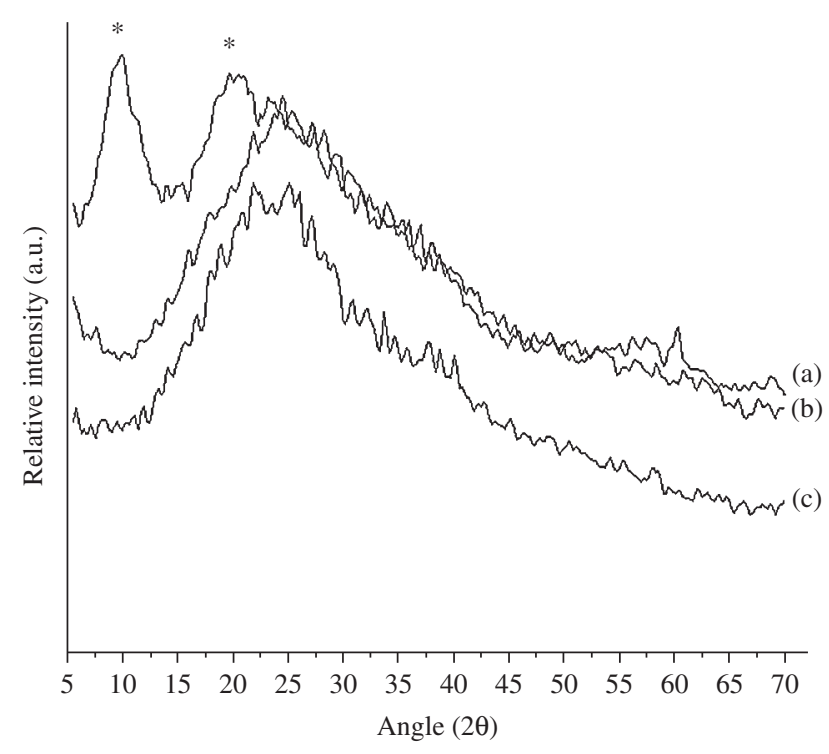

Figure 3. XRD of samples: a) lyophilized CyA, b) lyophilized PLGA 75:25 and c) lyophilized mixture.

mobility capacity among the polymeric chains, characterizing the observed amorphous state of lyophilized mixture.

\subsection{Thermogravimetry characterization}

The TG curves of PLGA 75:25 e CyA. The onset of mass loss for PLGA occurs at $247{ }^{\circ} \mathrm{C}$ and for CyA occurs at $254{ }^{\circ} \mathrm{C}$. In addition, the $100 \%$ mass loss occurs at $378{ }^{\circ} \mathrm{C}$ and $600{ }^{\circ} \mathrm{C}$ for PLGA $75: 25 \mathrm{e}$ CyA, respectively (Figure 4).

A thermal stability at $100-120^{\circ} \mathrm{C}$, temperature used in implants preparation, shows that both samples present thermal stability during the implant preparation, indicating the viability of the developed implant preparation method.

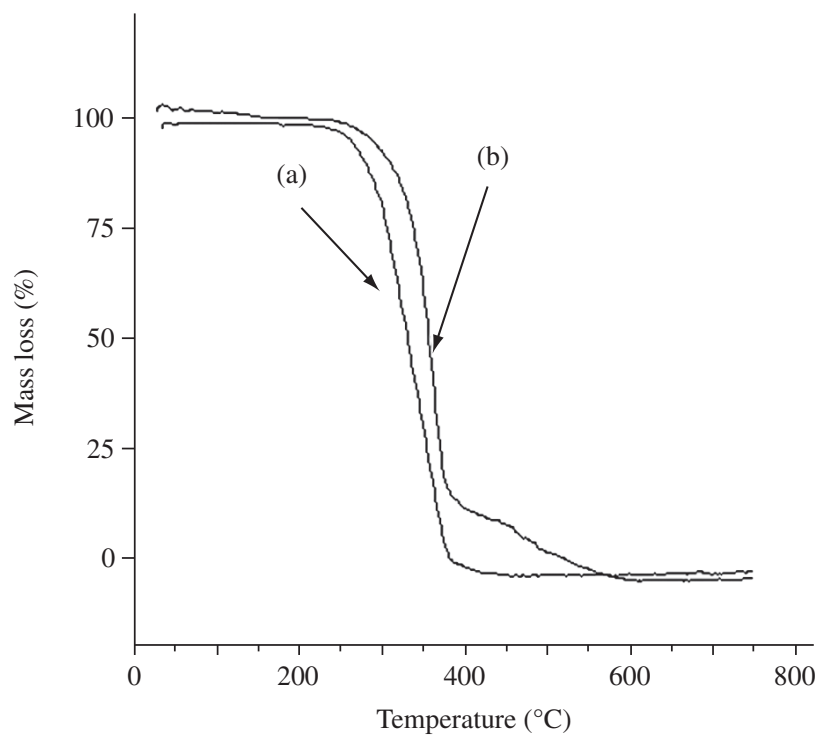

Figure 4. TG curves of samples: a) lyophilized PLGA 75:25; and b) lyophilized CyA.

\subsection{Differential scanning calorimetry characterization}

The glass transition temperature ( $\mathrm{Tg}$ ) of the polymer can be used as a measurement for the mobility of the macromolecules ${ }^{9}$ and the evaluation of the solid-to-liquid transition of the CyA characteristic consistent with a glass thermotropic liquid crystal ${ }^{10}$ also give information about the CyA molecular mobility and changes at its semi-crystalline state. These characteristics can be used to explain the presence or absence of physical interaction.

The evaluations of the glass transition temperature of the lyophilized drug and polymer precursors and in the systems were obtained from the DSC curves (Figure 5). 
In Figure 5 it is showed the estimated polymers glass transition temperatures $\left(50^{\circ} \mathrm{C}\right)$ for all analysed samples: lyophilized polymer (Figure 5a), lyophilized CyA (Figure 5b) and lyophilized mixture (Figure 5c). The CyA solid-to-liquid transition $\left(124^{\circ} \mathrm{C}\right)$ was detected only at the pure lyophilized CyA sample (Figure 5b). The changes verified in the Tg values of polymers and CyA solid-to-liquid transition were according to the literature ${ }^{10}$.The absence of variation in the Tg of the polymers in the pure PLGA 75:25 (Figure 5a) and lyophilized mixture (Figure 5c), samples indicates an unchanged mobility from polymers chains. These samples, either in the pure form or associated in systems, have presented the same capacity of chain mobility, leading to an indication of the absence of physical interaction between polymers and drug. The inexistence of the CyA solidto-liquid transition in the lyophilized mixture (Figure $5 \mathrm{c}$ ) is probably related to the rearrangement of drug molecular conformation into the polymeric matrix or maybe to its impossibility to be detected under the experimental conditions, result that confirms the XRD hypothesis. During the association of polymer to drug, no detectable change on chain and molecular conformation could be verified.

\subsection{The fourier transform infrared spectroscopy (FTIR) characterization}

The possibilities of chemical and physical interactions were also evaluated by FTIR (Figure 6). FTIR analysis was mostly based at the amide-I and ester region from the spectra once they are the specific chemical group present in each molecule under investigation, which would be a suitable indication for any chemical interaction that may occur among them. Also, the physical interaction between polymer network and drug was evaluated at the methyl asymmetric stretching region.

The FTIR spectra of the powders PLGA 75:25 and CyA with ratio 100:10 (Figure 6a) have given evidence of both PLGA (polymer) and CyA (drug) structure information, showing all major characteristic bands at $1750 \mathrm{~cm}^{-1}$ and $1628 \mathrm{~cm}^{-1}$ correlated to the presence of amides and ester groups in CyA and PLGA 75:25, respectively. These results are endorsed by FTIR spectra obtained from both pure CyA and PLGA 5:25 (data not showed) and with the previously reported literature ${ }^{4,11}$.

The lyophilized mixture (Figure 6b) presented similar vibrational band at $1740 \mathrm{~cm}^{-1}(\mathrm{C}=\mathrm{O}$ ester stretching $)$ and band at approximately $1630 \mathrm{~cm}^{-1}(\mathrm{C}=\mathrm{O}$ primary amide stretching $)$. On the other hand, im-

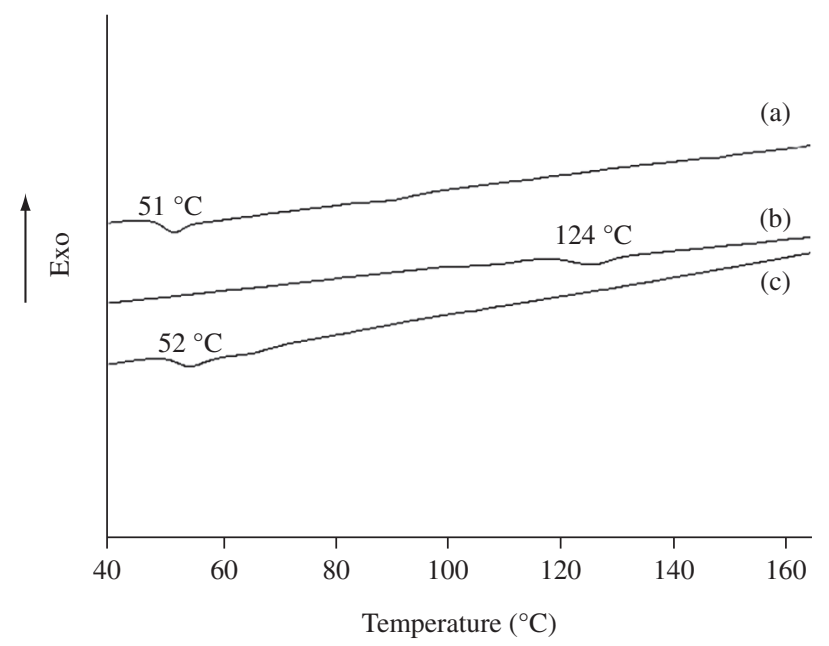

Figure 5. DSC curves of the samples: a) PLGA 75:25 lyophilized; b) lyophilized CyA and c) lyophilized mixture PLGA 75:25. portant bands related to $\mathrm{vC}-\mathrm{H}$ stretching were found to be slightly different at 2959 and $2924 \mathrm{~cm}^{-1}$ when compared to both precursors powders (2944 and $2995 \mathrm{~cm}^{-1}$ ). Peaks at 3319 and $3316 \mathrm{~cm}^{-1}$ associated with $v \mathrm{C}-\mathrm{N}$ stretching of amide-I were identified in the samples from lyophilized mixture (Figure 6b).

The characteristics bands of amide-I and ester groups in the region of $1680-1630 \mathrm{~cm}^{-1}$ and $1725-1750 \mathrm{~cm}^{-1}$ have given some evidence of the lack of any major strong chemical interaction. Besides that, the integrity of amide-I stretching band $(\mathrm{C}-\mathrm{N})$ indicates the stability of $\beta$-sheets intermolecular interaction present in the drug and, consequently, CyA stability and biological activity was maintained ${ }^{11}$. Although the presence of a minor change in the methyl stretching band in polymer/drug samples, we have not considered this value as associated with some physical interaction. Such results are attributed to some probable alteration on CyA conformation, endorsed by the presence of N-H amide-I stretching. The observed high intensity band associated with $\mathrm{N}-\mathrm{H}$ amide-I stretching in all investigated samples are likely to be due to a CyA molecular rearrangement through hydrophilic interaction aiming to reach the most stable thermodynamic conformation at polymer/drug interface, supporting the results from XRD and DSC techniques.

TG curves have proved the thermal stability of samples at the implant preparation temperature. Moreover, the XRD patterns associated with DSC and FTIR results showed the drug distribution into the polymeric matrix.

In FTIR spectra no extra bindings or chemical shifts were observed, indicating that there is no strong chemical interaction between polymer and drug into the polymer/drug network. In addition no detectable changes in polymer thermal behavior from DSC data are attributed to the absence of physical interactions between polymer and drug. These results confirm the drug chemical stability, the permanence of their biological activity and the possibility of drug sustained delivery system profile.

\subsection{The preliminary in vitro studies}

The preliminary in vitro release study of the intraocular implant developed was performed to determinate the initial CyA delivery profile. A slow and prolonged release was observed during the 23 weeks (Figure 7).

The cumulative CyA released was $8.4 \%$ to mixture lyophilized implants (Figure 7). The ratio of CyA released is most probably

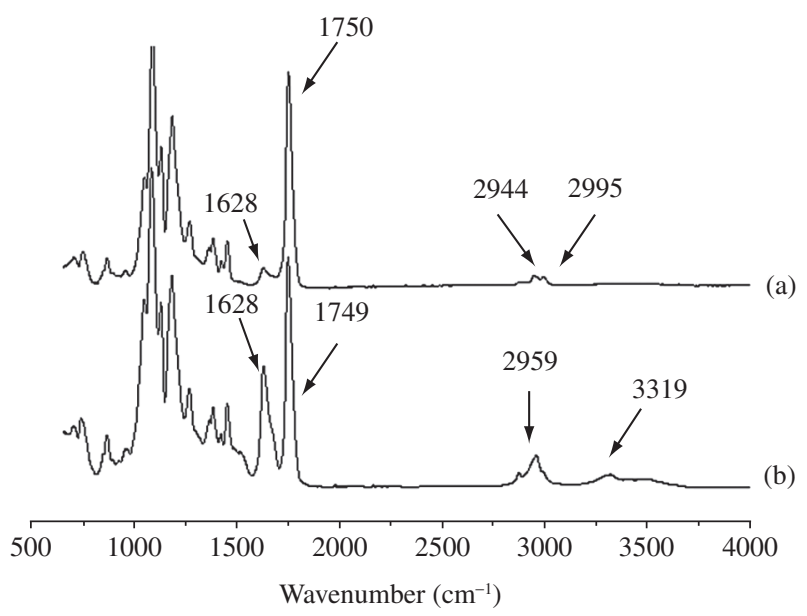

Figure 6. FTIR spectra in the role wavelength range $\left(1500-4000 \mathrm{~cm}^{-1}\right)$ of the samples: a) powders PLGA 75:25 and CyA in ratio 100:10, respectively and b) mixture lyophilized. 


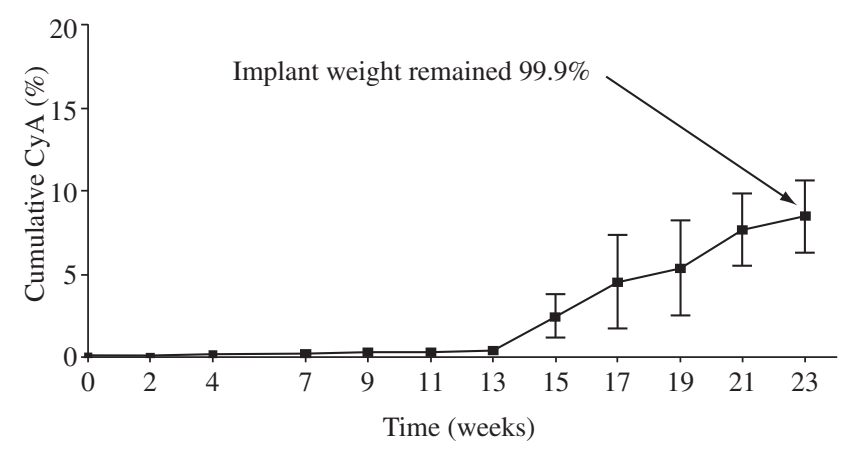

Figure 7. Cumulative release of CyA from: mixture lyophilized implants in PBS buffer 7.4. The values are showed as mean \pm SD of $n=3$.

regulated by the diffusion of the drug in the swelled polymer network and not by the polymer matrix disintegration. Such affirmation can be made once it was not observed any significant implant mass loss during the in vitro release assay (Figure 7). Besides that, the drug release profile can be also attributed to the homogeneous implant surface obtained as showed in SEM micrograph (Figure 2). Consequently, such results indicate the possibility of the biodegradable implants to deliver CyA in a prolonged way when incubated in similar posterior segment of the eye conditions.

\section{Conclusions}

In the present work biodegradable polymeric intraocular implants were developed and obtained from lyophilized mixture sample. Based on the nanostructure evaluation of the polymer/drug network, we have successfully characterized the system with several different techniques. We have showed by XRD the CyA distribution into the implant polymeric matrix. These findings were confirmed by DSC and FTIR results. Besides that, DSC and FTIR showed the absence of some detectable physical and chemical interaction between drug and polymer and the presence of the CyA in structural biological activity. The morphological characterization showed the homogeneous implant and the preliminary in vitro release studies proved the implant capacity to release the drug in a prolonged profile when incubated into a medium condition similar to the eye constituents. In summary, the delivery system developed and fully characterized in the present work can be applied, in the future, for the treatment of posterior uveitis.

\section{Acknowledgements}

The authors wish to thank Prof André Faraco by assisting in sample preparation and laboratory protocols. This work was financially supported by CNPQ/FAPEMIG/CNPq.

\section{References}

1. Merkli A, Tabatabay C, Gurny R, Heller J. Biodegradable polymers for the controlled release of ocular drugs. Prog. Polym. Sci. 1998; 23(3):563-580.

2. Yasukawa MDT, Kimura H, Tabata Y, Ogura Y. Biodegradable scleral plugs for vitreoretinal drug delivery. Adv. Drug. Del. Ver. 2001; 52(1):25-36.

3. Avitable T, Marano F, Castiglione F, Bucolo C, Cro M, Ambrosio L, et al. Biocompatibility and biodegradation of intravitreal hyaluronan implants in rabbits. Biomaterials 2001; 22(3):195-200.

4. Stevenson C, Tan MM, Ballesteros DL. Pharmaceutical liquid crystals: The relevance of partially ordered systems. J. Pharm. Sci. 2003; 94(9):1861-1880.

5. Fialho SL, Cunha-Júnior AS. Manufacturing techniques of biodegradable implants intended for intraocular application. Drug. Deliv. 2005; 12(2):109-116.

6. Kunou N, Ogura Y, Yasukawa T, Kimura H, Miyamoto H, Honda Y, Ikada Y. Controlled intraocular delivery of ganciclovir with use of biodegradable scleral implant in rabbits. J. Cont. Rel. 1995; 37:143-150.

7. Yasukawa T, Kimura H, Tabata Y, Ogura Y. Biodegradable scleral plugs for vitreoretinal drug delivery. Advanced Drug Delivery Reviews 2001; 52(1):25-36.

8. Ballesteros DL, Fattah AA, Stevenson CL, Bennet DB. Properties and stability of a liquid crystal form of cyclosporine-the first reported naturally occurring peptide that exists as a thermotropic liquid. Journal of pharmaceutical Science 2003; 92(9):1821-1831.

9. Elkharraz K, Faisant N, Guse C, Siepmann F, Arica-Yegin B, Oger J.-M, Gust R, Goepferich A, Benoit JP, Siepmann J. Paclitaxel-loaded microparticles and implants for the treatment of brain cancer: preparation and physicochemical characterization. International Journal of Pharmaceutics 2006; 314(2):127-136.

10. Chan HK, Clark AR, Feeley JC, Kuo MC, Lehrman SR, Pikal-Cleland K, Miller DP, Vehring R, Lechuga-Ballesteros D. Physical stability of salmon calcitonin spray-dried powders for inhalation. J. Phar. Sci. 2005; 93(3):792-804.

11. Jayasuriya AC, Assad M, Jayatissa AH, Ebraheim NA. Dissolution behavior of biomimetic minerals on 3D PLGA scaffold. Surface and Coating Technology 2006; 200(22-23):6336-6339.

12. Dong X, Shi W, Yuan G, Xie L, Wang S, Lin P. Intravitreal implantation of the biodegradable cyclosporin A drug delivery system for experimental chronic uveitis. Graefe's Arch. Clin. Exp. Ophthalmol. 2006; 244(4):492-497. 